\title{
Understanding the Evolution of Circular Economy through Language Change
}

\author{
Sampriti Mahanty ${ }^{1}$, Frank Boons ${ }^{2}$, Julia Handl ${ }^{3}$, Riza Theresa Batista-Navarro ${ }^{4}$ \\ Alliance Manchester Business School, The University of Manchester ${ }^{1,2,3}$ \\ School of Computer Science, The University of Manchester ${ }^{4}$ \\ sampriti.mahanty@postgrad.manchester.ac.uk ${ }^{1}$ \\ \{frank.boons, julia.handl, riza.batista\}@manchester.ac.uk ${ }^{2,3,4}$
}

\section{Introduction}

Drawing on the work of Thomas Kuhn, philosophers of science have developed understandings of the process of scientific development (Campbell, 1974b; Popper, 1972; Toulmin, 1967, 1972; Hull, 1988) using metaphors relating to Darwin's theory of natural selection. The consequence of this is the conceptualization of scientific development as constituting an "evolutionary process". The defining feature of adopting an evolutionary orientation in understanding scientific development is suggesting an analogy between the process of biological evolutionary process and that of gain in knowledge (Bradie, 1986). According to this perspective, in the course of evolution, species become more adaptive to their natural environment by undergoing natural selection. Likewise, scientific progress is a result of selection mechanisms on an individual level and in the scientific community.

Scientists are the "central subjects" in the process of scientific development since they are the entities who read the literature, perform experiments, publish the results and pass on knowledge. Textbooks, journal articles are "vehicles" in this process of scientific and conceptual development. Scientists then go on to form communities based on common cognitive, social and philosophical grounds (Hull, 1988).

While the production of scientific knowledge is an outcome of selectionist mechanisms, it is closely related to evolution of human language
(Popper, 1984; Bradie, 1986). Scientific communities tend to use the same umbrella terms ("lexicon kind-terms") at the least, having meanings that are shared and understood by members of such communities. Language thus becomes a crucial indicator to assess the shift or development in ideas (Kuhn, 1990).

For our study, we propose to focus on a specific concept - that of Circular Economy (CE). The evolutionary perspective of understanding scientific development is the framework that we implement to understand this concept. While this forms a part of our larger ongoing work to determine the process of emergence, diffusion and evolution of the concept of 'circular economy' in academic discourse, the focus of this study is to understand the evolution of $\mathrm{CE}$ from the lens of language change, as language can be a crucial indicator of the shift in ideas in a particular scientific field.

There are two aspects of language change that can be investigated. The first one is lexical change, i.e., the generational shift in the representation of words over time, and the second is semantic change, i.e., the evolution of word usage, sometimes to the point that the modern meaning is radically different. In some cases, the semantic change that words undergo happens by means of acquiring additional meanings, rather than original meanings becoming outdated or being replaced. In this study we will be analysing how the language used in academic discussions pertaining to $\mathrm{CE}$ has changed semantically. It is worth noting that 
the meaning and central theme of this concept has remained the same; however, we hypothesise that it has undergone semantic change by way of additional layers being added to the concept.

\section{Circular Economy}

Circular Economy (CE) refers to a system of provision in which resources are circulated between production and consumption rather than linearly transformed from production to consumption to waste. It has gained immense traction amongst academics, practitioners and policy-makers for its perceived capacity to operationalise Sustainable Development ${ }^{1}$ : (Geissdoerfer et al., 2017). It is only in the last 15 years that it has emerged as a field in its own right, being referred to directly and independently in the academic literature, distancing itself from the antecedent fields (e.g., industrial ecology) (Prendeville et al., 2018).

The diffusion of CE has become prominent since 2015 as indicated by the steep increase in the number of academic articles published (Appendix Figure 1). This can be attributed to the fact that more recently, $\mathrm{CE}$ has gained dominance in literature discussing issues related to sustainability. While $\mathrm{CE}$ has its roots in Industrial Ecology focused on industrial processes, pollution etc., nowadays $\mathrm{CE}$ is actively being referred to in the context of micro-level interventions for sustainable development, e.g., circular product design, circular business models (Bocken et al., 2016). The contemporary understanding of $\mathrm{CE}$ and its practical applications to industrial processes and economic systems has evolved to incorporate different features and contributions from concepts beyond $\mathrm{CE}$ which share the theme of closed loops (Geissdoerfer et al., 2017). This kind of change in context makes CE suitable as the focus of our study in scientific concept evolution.

\section{Methodology and Findings}

The first step towards the analysis of language for detecting semantic change w.r.t $\mathrm{CE}$ is the collection of academic literature on $\mathrm{CE}$ from the

\footnotetext{
${ }^{1}$ Development that meets the needs of the present without compromising on the ability of future generations to meet their own needs
}

Scopus database ${ }^{2}$. Using the query "circular economy", we retrieved a total of 3,300 scientific papers. For the pre-processing of the corpora, we performed word stemming and the removal of stop-words, extraneous white spaces and punctuations. We conducted topic modelling using the Latent Dirichlet Allocation algorithm (Blei et al., 2003) on the academic abstracts by using the topic models3 package available in $\mathrm{R}$. The number of topics (K) was fixed at 20 , based on the examination of coherence scores 4 of the topic model coupled with manual analysis. The results from the topic model were plotted to visualize the topics over time. Based on the results from the topic model we noticed a structural change in academic discussions pertaining to $\mathrm{CE}$ in the year 2015 (Appendix Figure 2). There was a significant change in the proportion of topic distribution before and after 2015. Based on this finding, two datasets were formed: an early set consisting of papers published from 2006 to 2014, and a contemporary CE set composed of papers published from 2015 to March 2019.

We investigated and compared the CE literature across the early and contemporary data sets using two approaches to analyse semantic change in language over time. First is the development of co-occurrence networks based on keywords associated with the documents. Nodes of the network correspond to the keywords (with a node for $\mathrm{CE}$ as the centroid), and edges indicate the co-occurrences. A cooccurrence network using the bibliometrix package $^{5}$ in $\mathrm{R}$ was generated based on each of the early (Figure 1) and contemporary (Figure 2) data sets. We observed that there is a significant difference between the two co-occurrence networks. Contemporary CE literature was found to be more strongly linked to "business models", "supply chain", "product design". Meanwhile the focus of early CE literature was more on "ecology", "industrial economics" and

\footnotetext{
${ }^{2}$ https://www.scopus.com/search/form.uri?display=basic

${ }^{3}$ https://cran.r-

project.org/web/packages/topicmodels/topicmodels.pdf

${ }^{4}$ The coherence score is for assessing the quality of the learned topics. For one topic, the words $\mathrm{i}, \mathrm{j}$ being scored in $\sum \mathrm{i}<\mathrm{jScore}$ (wi, wj) have the highest probability of occurring for that topic.

${ }^{5}$ http://bibliometrix.org
} 
"environmental management". These observations confirm that the concept of CE has undergone some change over the years that are reflected by a shift in focus in the context of its application (as discussed in Section 2). We note that despite this expansion, the core meaning of the concept has not changed over time (as evidenced by the nodes that are common between the two networks, e.g., "sustainable development, "waste management", "recycling".

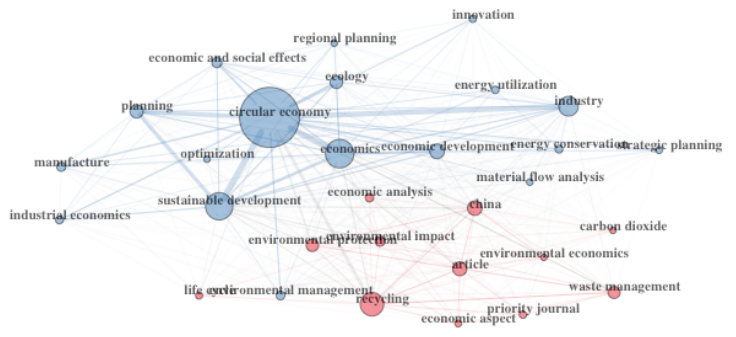

Figure 1: Co-occurrence network drawn from the early dataset (2006-2014)

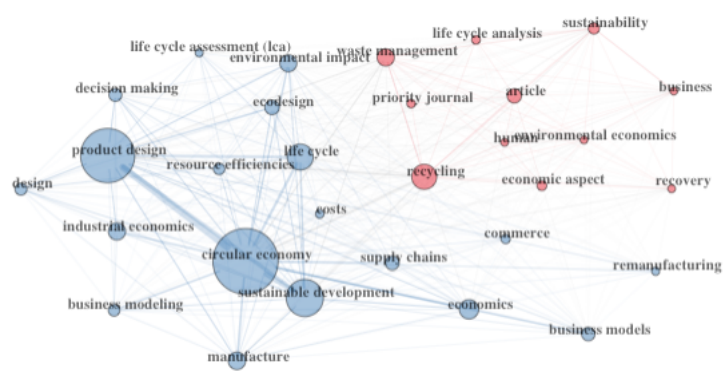

Figure 2: Co-occurrence network drawn from the contemporary dataset (2015-2019)

Our second method is underpinned by the development of word embedding vectors (Bojanowski et al., 2017). We obtained word embeddings using gensim's word $2 \mathrm{vec}^{6}$ implementation on the full text of academic articles. The word embeddings were trained on each of the two data sets i.e., early and contemporary $\mathrm{CE}$ literature. We then compared the word embeddings based on the target word of interest "circular economy" across the two time periods. The cosine similarity between word embedding vectors across the two time frames is only 0.195 . To better understand the similarity measure between the word embeddings we conduct a random sampling on each of the early and contemporary datasets to create two subsets out of each, then trained embeddings on each of the subsets. The cosine similarity between each of the subsets came across to be quite high i.e., 0.62 and 0.743 in the early and contemporary dataset respectively. This provides us with the basis of comparing the cosine similarities and we can conclude that the cosine similarity of word vectors between the late and early dataset is considerably low at 0.195 .

If we examine the nearest neighbours of CE (i.e., words with highest similarity to CE) from the two time periods in Table 1, we see a shift from the environmental and industrial focus to a perspective, which integrates an approach, which is innovation, business focused and incorporates the social dimension of $\mathrm{CE}$ as well. The results from the word embeddings conform to the results from the co-occurrence networks. The early literature primarily address macro-level themes in the context of environmental management and industries while the contemporary literature focuses on more micro- level interventions like business models, product design and supply chain.

\begin{tabular}{|c|c|}
\hline Early dataset & Contemporary dataset \\
\hline Resource & Innovation \\
Materials & Business Models \\
Recycling & Social \\
China & Strategies \\
Environmental & Companies \\
Economic & Supply Chain \\
Industrial & Sustainable \\
& development \\
\hline
\end{tabular}

Table 1: Closely associated word vectors to CE for each of the early and contemporary datasets, ordered by decreasing similarity.

\section{Conclusion}

We have discussed that semantic change in language is a reflection of shifts in scientific ideas, which in turn help explain the evolution of a concept. This helps us to build an understanding of conceptual evolution. This forms a part of our ongoing work to understand the concept of $\mathrm{CE}$ starting from the emergence of the concept, to its adaption, diffusion and evolution. 


\section{References}

Bocken Nancy, Pauw Ingrid de, Bakker Conny \& Grinten Bram van der. (2016). Product design and business model strategies for a circular economy, Journal of Industrial and Production Engineering, 33:5, 308-

320, DOI: $10.1080 / 21681015.2016 .1172124$

Bojanowski, Piotr, Grave Edouard, Joulin Armand, and Mikolov Tomas (2017). Enriching word vectors with subword information. Transactions of the Association for Computational Linguistics: 135-146.

Blei, D.M.; Jordan, M.I.; Ng, A.Y. (2003) Latent dirichlet allocation. Journal of Machine Learning. Res. 2003, 3, 993-1022.

Bradie, Michael. "Assessing evolutionary epistemology. (1986)" Biology and Philosophy 1.4: 401-459.

Campbell, Donald. T. (1974b). "Evolutionary Epistemology”. In: Schlipp, P. A. (Ed.), The philosophy of Karl Popper. LaSalle, IL: Open Court, 413-63.

Geissdoerfer, Martin \& Savaget, Paulo \& Bocken, Nancy \& Hultink, Erik. (2017). The Circular Economy - A new sustainability paradigm? Journal of Cleaner Production. 143. 757-768. 10.1016/j.jclepro.2016.12.048.

Hull, David L. (1988). Science as a Process an Evolutionary Account of the Social and Conceptual Development of Science. University Of Chicago Press.

Kuhn, Thomas S. (1990). The Road since Structure. PSA: Proceedings of the Biennial Meeting of the Philosophy of Science Association_1990:3-13.

Popper, Karl. R. (1972) (1979). Objective Knowledge: An Evolutionary Approach, Oxford: The Clarendon Press

Popper, Karl R, and William Warren Bartley. (1984) "Postscript to the logic of scientific discovery."

Prendeville, Sharon, Emma Cherim, and Nancy Bocken. (2018). Circular cities: mapping six cities in transition. Environmental innovation and societal transitions. 26 171-194.

Toulmin, Stephen. (1967). "The Evolutionary Development of Natural Science", American Scientist 55, 456-471

Toulmin, Stephen. (1972). Human Understanding: The Evolution of Collective Understanding, Volume 1. Princeton, NJ: Princeton University Press.

\section{Appendices}

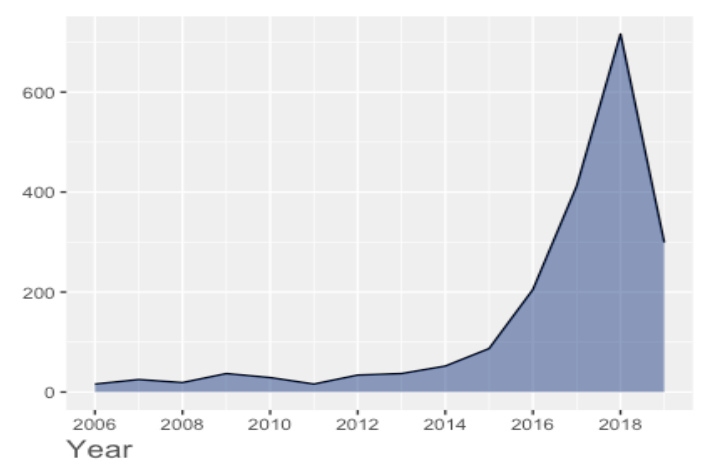

Figure 1: Production of academic articles on $\mathrm{CE}$

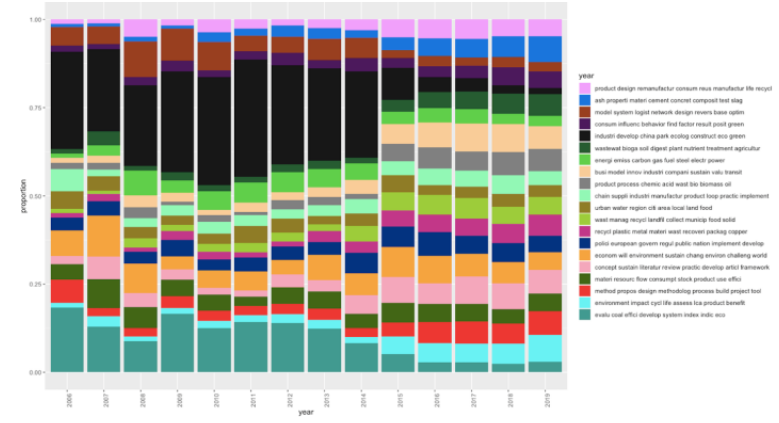

Figure 2: Proportion of topic distribution over time 